\title{
Richard D. Klausner
}

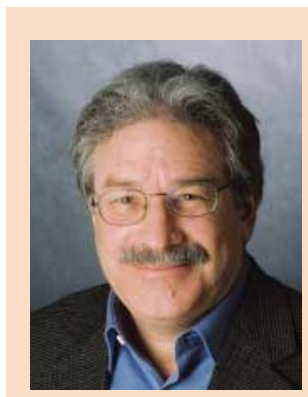

Executive Director, Global Health, Bill \& Melinda Gates Foundation, PO Box 23350, Seattle, Washington 98102, USA

Richard D. Klausner is Director of the Global Health programme of the Gates Foundation, which aims to improve global health equity by forging global collaborations and funding R\&D. Klausner previously served as Director of the National Cancer Institute $(\mathrm{NCl})$, a post in which he oversaw the administration of the second largest HIV/AIDS programme in the world, and co-founded and developed the Vaccine Research Center. After postgraduate medical training at Harvard, he began his research career at the National Institute of Child Health and Human Development. He has held several advisory roles to the US government, was Senior Fellow at the National Academies of Science and is a past president of the American Society for Clinical Investigation.

Existing global health initiatives have made limited progress. How can the Gates Foundation make a difference? There has been a huge lack of attention to the development of new tools and technologies specifically for diseases of the poor, especially tuberculosis and malaria. Insufficient engagement in discovery and development for global diseases stems from the perceived and real failure of the marketplace in developing countries. The Gates Foundation can only partially address that inequity of attention and we cannot, and should not, do it alone. Instead we intend to forge public-private partnerships and stimulate attention by investing in $\mathrm{R} \& \mathrm{D}$.

How has working at the NCI equipped you for leading a global public-private venture? The NCI had traditionally been the NIH institute with the most experience in product development and translating the development of drugs to changing practice, so it's been incredibly helpful for me. However, the Gates Foundation is not a funding entity for supporting a large research community. It's fantastic that the NIH and others do that because we can build on it, but we will focus more on problem solving and funding fewer, larger projects.

\section{One such project is the Global HIV Vaccine} Enterprise. How will you overcome the technical challenges of developing an HIV vaccine? We engaged in a lengthy discussion with HIV research groups worldwide to evaluate where we are in the development of an HIV vaccine. We need an overarching approach to ensure that $H I V$ vaccine $R \& D$ is optimized towards maximizing what we learn and rationalizing our ideas. For example, have we been developing ways of measuring the immune response that are reproducible? Can we accurately compare different sets of immunogenicity trial data? This requires resources and infrastructure that individual grants will never support. The fragmented system that is great for discovery is not optimized for development, so we need to link the two processes. We're attempting to bring the rigour of scientific design not only to individual experiments, but to the entirety of the approach.

\section{What key steps will you take to globally} standardize and improve HIV research? By summer 2004, a steering committee will have formed a strategic plan based on reports from many HIV research groups that will be used to coordinate funding so that provisions that are currently rate-limiting are made available. Not all of the resources can come from the Gates Foundation - we need a global recommitment to the HIV vaccine, which we believe is the single most important medical technology that the world needs to deal with this global pandemic. Difficult behavioural and sociological changes are also necessary to enable this kind of cooperation and integration while maintaining competition and openness to new ideas.

You previously cited "a lack of political will and social conscience in the US" as a fundamental barrier to global healthcare. How might the Gates Foundation address this problem? First, by contributing to $R \& D$ costs we make it more likely that products that are needed are created. Second, we provide grants — such as the Global Alliance for Vaccines and Immunizations (GAVI) fund - that create a new and successful model for dealing with the issues of market failure in the developing world, by increasing supply and demand for vaccines, increasing competition between vaccine producers and reducing prices. Eight million children a year in the developing world are now immunized because of this new global business mechanism. But we need to look at similar changes for reproductive health commodities, drugs and diagnostics. Global health is much more on the front burner than it was five years ago, and that's an important part of the optimism required for success.

How will the resources of the Gates Foundation be split geographically, and between R\&D, infrastructure and healthcare?

The vast majority of resources will go to $\mathrm{R} \& \mathrm{D}$ to create new products, and this will certainly not be limited to the United States or the developed world. A significant amount will also fund mechanisms that improve the effectiveness and efficiency of global access. But let me emphasize that we do not pay for medical care or build hospitals and clinics: that is the responsibility of the public sector.

But without necessary infrastructure how can your initiatives really have any impact? Well, the GAVI fund is really a great example of where we're bringing together the public and private sectors, and creating a pathway of rationalizing global procurement and, consequently, vaccine delivery has been successful.

What single thing would you like to have achieved at Global Health by the end of 2004? What I'm very excited about is the launching of a series of initiatives that run the gamut of public health intervention. One example is the large programme in India studying whether we can rapidly intervene to prevent the current local AIDS epidemic spreading to a more generalized epidemic.

\section{Would you like to speculate on when we might} have an HIV vaccine?

A field can always be lucky, but we're all very sober about how long it's going to take and how hard it's going to be. 